\title{
Intelligent and Adaptive Tutoring for Active Learning and Training Environments
}

Claire Kenny and Claus Pahl ${ }^{1}$

School of Computing, Dublin City University, Dublin 9, Ireland

\begin{abstract}
Active learning facilitated through interactive and adaptive learning environments differs substantially from traditional instructor-oriented, classroom-based teaching. We present a Web-based e-learning environment that integrates knowledge learning and skills training. How these tools are used most effectively is still an open question. We propose knowledgelevel interaction and adaptive feedback and guidance as central features. We discuss these features and evaluate the effectiveness of this Web-based environment, focusing on different aspects of learning behaviour and tool usage. Motivation, acceptance of the approach, learning organisation and actual tool usage are aspects of behaviour that require different evaluation techniques to be used.
\end{abstract}

\section{Keywords}

automated tutoring; correction; personalisation; feedback; guidance; skills training; database programming.

\section{Introduction}

Intelligent and adaptive computer-based tutoring is being pursued for more than 40 years (Hartley, 1973; Patel, Scott, \& Kinshuk, 2001). Teaching modes focusing on behavioural control, discovery-type learning and teaching through rational argument have been implemented using computer-based tutoring, simulation and teaching of problem-solving skills. Question and answer-based conversations have always played a major role (Scott, 2001). This earlier work was seminal and pioneering in understanding learners as being active constructors of knowledge (Laurillard, 1993). We now have the technology to implement intelligent and adaptive systems as practical solutions (Grabinger, 1996). We realise that skills and knowledge are best acquired within realistic contexts that allow learners to actively participate in knowledge construction and skills training (Nardi, 1997).

In this paper, we refer to a specific context in particular - computing education. In contexts such as computing, engineering, science or medicine, the combination of conceptual knowledge and practical skills is often of paramount importance. In computing, execution models and abstract software development paradigms on the one hand and programming on the other hand are examples that illustrate this point. We can often distinguish between classical conceptual knowledge-oriented learning and skills-oriented training.

We present an interactive learning and training environment with an automated, adaptive tutoring system at the core (Kenny, 2006). This environment supports the SQL (Structured Query Language) part of a database courseware system. It aims to provide students with an environment that integrates a range of aspects needed to become a knowledgeable and skilled database programmer. After initially too

\footnotetext{
${ }^{1}$ Corresponding author. Email: Claus.Pahl@dcu.ie
} 
restricted tutoring systems, the focus of researchers has shifted towards more flexible adaptive hypermedia systems to allow the learner a higher degree of control (De Bra Brusilovsky, \& Houben, 1999; Brusilovsky, 2000). Instructional design for these types of active learning environments is still an area of active research and the learner behaviour in these systems is not well understood (Murray, 1999). Our aim is to demonstrate that, to be effective, active learning using an automated tutor requires scaffolding support in the form of immediate and meaningful high-level feedback and personalised guidance (Ravenscroft, Tait, \& Hughes, 1998; Winnips, 2001; Chou, Chan, \& Lin, 2002).

We present and evaluate an intelligent and adaptive tutoring system for database programming that supports active learning and skills-oriented training. At the core is an analysis and correction feature that provides immediate, knowledge-level feedback

to the student for solutions submitted to the system. The system also provides studentcontrolled adaptive guidance across a range of problems based on an accumulative analysis of the errors a student makes. We describe the environment and its objectives and discuss the relationship of the instructional design that the environment supports to theoretical principles. We address the students' learning behaviour and discuss the effectiveness of the environment with its specific active learning and training support.

\section{Active learning and training - environment, principles and objectives}

\subsection{An online database programming environment}

The interactive SQL learning and training tutor is embedded into an online courseware system for a second year undergraduate database course. The SQL section forms a central component of this course, as database programming is a core learning objectives. Database programming (i.e. defining, updating, and querying database tables) is a skill that requires training on the part of the student. Database programming in SQL also requires conceptual understanding of the underlying data model with its structures, operations, and constraints. The environment provides different features for the different aspects of SQL programming:

- Conceptual knowledge. Conceptual knowledge is presented in a virtual lecture system based on an audio-visual presentation.

- Procedural knowledge. SQL and its underlying data model are about the execution of instructions. SQL is a language that implements database definition and manipulation operations. Procedural knowledge is presented in an animated tutorial system that illustrates the execution of these operations in a step-by-step fashion.

- Programming skills. SQL programming is the core activity, supported by an interactive tutorial that guides the student through exercises to be worked on within the system. SQL queries are often complex and difficult to formulate.

\subsection{An interactive database programming tutor - design principles}

The interactive tutorial is a focal element of this investigation; see Fig. 1 for an architectural overview. The design of this feature is guided by objectives derived from teaching and learning best practises and research into online learning: 
- Learning-by-doing and active learning. Active learning is a successful way of learning practical tasks (Grabinger, 1996). Students can actively create and execute programming solutions. This form of procedural knowledge generation leads to a richer and more successful learning experience. Active construction of knowledge and skills results in an increased ownership of the student in the learning process.

- Realistic setting. We replicate the real-world situation of database programming by having students develop their solutions in a realistic setting and execute these. A realistic setting improves the learning experience and demonstrates the applicability of knowledge and skills (Herrington \& Oliver, 2000).

\section{$<<$ Figure 1 goes here $>>$}

Our proposal is that active online learning and training in a realistic setting should be combined with an automated tutor that provides immediate feedback and adaptive guidance (Melis \& Ullrich, 2003). Guidance and feedback provide instructional support in the environment.

- Error analysis and categorisation. We have analysed the SQL language in order to assess aspects of SQL statements a typical student might have difficulty with. The course instructor's experience has been an additional input into this process. Any error made by a student is associated with an error category. We use a multilevel error categorisation scheme in order to address the multiple roles an errorcausing construct can play. The correction component analyses a submitted SQL solution based on the syntax and semantics of the language. It considers typical errors and categorises the student errors appropriately.

- Immediate local feedback. This type of feedback is based on an analysis of the correctness of student submissions and actually executed correct answers. It is important in a formal language tutoring system to provide feedback on the correctness of a submission. The results of the analysis and correction method can influence the scaffolding used to support a student. Errors made need to be contextualised.

- Guidance based on personalised assessment. Mid-to-long term guidance based on a personalised assessment needs to be offered. This global feedback consists of categorised and ranked errors and difficulties, based on an accumulative diagnosis of student performance across a range of problems. Students are offered a menu of further questions to practise, based on the type and amount of errors.

- Adaptive tutoring. Adaptivity techniques for use in this knowledge-based feedback and guidance system is another central aspect. It allows us to adapt the student's learning experience based on specific preferences chosen by the student as well as the context in which the student is based. Adaptivity techniques include changing both content and navigation options. We use both of these techniques to determine the type of immediate feedback and long-term guidance the student will receive based on information about his previous actions.

Technical solutions that implement the tutor for domain-specific activities, such as database programming here, are crucial for providing the necessary quality of feedback and personalisation. Therefore, solution analysis and the mapping from error categories to student performance profiles play a central role in making the approach effective.

\subsection{An interactive database programming tutor - pedagogical setting}


Adaptive feedback and guidance specific to the submitted solution and also the student performance are important scaffolding elements for active, skills-oriented learning and training (Guzdial \& Kehoe, 1998). The system needs to include a multilevel feedback approach in order to, firstly, gradually increase and fade the feedback given and, secondly, to allow a student to choose the levels of feedback and guidance provided. A system default starts at a low level, increases the level gradually, and then begins to fade the feedback after a time. This needs to be dependent on the overall performance across a range of problems. The apprenticeship theory, situated within the constructivism paradigm, is particularly suited to skills training (Collins, Brown, \& Holum, 1991). An important feature of this theory is scaffolding, of which feedback is a classic example.

Active learning plays an important role in recent instructional design approaches. Learning is seen as a process in which learners actively construct knowledge and acquire skills. In the context of computer-supported interactive learning and training environments, the role of this computer environment needs to be defined. An environment, such as the SQL programming environment, is a tool that mediates the interaction between learner and content. Activity theory is a conceptual framework that can describe the structure, development, and context of computer-supported activities (Engeström, 1987; Nardi, 1997). Conversation theory specifically addresses the use of learning technologies for online learning (Scott, 2001). The emphasis on the interactions and conversations between learners and their environment explains the principle of tool mediation. It highlights the importance of knowledge-level interaction, i.e. interaction in terms of SQL programming problems and solutions.

\subsection{Experiment and objectives}

Our aim is to demonstrate that a student can be engaged in solving problems in an activity-based, realistic setting. Database application development provides a meaningful problem. The database courseware system creates a realistic setting by integrating tools into a learning and training environment that resembles tools of a real database development environment.

These active learning and training features are enhanced by the inclusion of instructional functionality (guidance and feedback). Our hypothesis is that active learning and skills training, if provided in an autonomous environment where the human tutor is not always available, needs to be supported by intelligent, knowledgelevel feedback and adaptive guidance through an automated tutor.

We have built an automated tutoring system for SQL language learning and training; see Fig. 1. A correction technique based on the analysis of a student solution structure is at the core of this system. The system includes domain-specific feedback on the correction results and personalised guidance features.

- Error analysis and categorisation. Our aim is to demonstrate the possibility of providing immediate and automated feedback for student at knowledge-level (Ravenscroft et al., 1998). Our technique analyses and corrects student solutions to problems that are formulated in a formally defined language. This formal definition allows automated analysis. This feedback needs to be sufficiently 
comprehensive and accurate. Accuracy refers to the degree of correctness of system analyses.

- Adaptive tutoring. Our aim is to integrate adaptive guidance (Brusilovsky, 2000) into an intelligent feedback system in order to provide a student with a summative evaluation and a targeted set of problems for further study. A domain-specific, facetted categorisation is the basis of this diagnosis and recommendation approach. Constructive support for students can address problems that are difficult for them. We aim to achieve this through a system that can adapt the feedback and recommendation to the specific situation of individual students.

Programming activities are at the centre of our learning and training environment. However, supporting the learner through scaffolding is essential from the instructional perspective (Collins et al., 1991). In addition to just mediating between student and database tools, the environment must fulfil functions of the instructor. These instructor functions are based on the aforementioned analysis and mapping techniques.

- The environment replaces the instructor in form of a virtual master that guides a learner through exercises and that provides immediate feedback on activities.

- Each activity needs to be complemented by links to the background (conceptual and procedural knowledge in form of virtual lectures and animated tutorials) relevant and problem-related for the activity in question.

We aim to demonstrate that the automated tutor can replace some core functions of the human tutor.

\section{Evaluation method}

The tutoring system has been used in an undergraduate module in a computing degree. The system properties and its underlying pedagogical principles are the basis for its evaluation. We use three separate evaluation methods to carry out the evaluation (Kinshuk, Patel, \& Russell, 2000):

- Student Performance. Student performance is a substantial part of any degree programme, reflected in exam results. We compare the results obtained by current students compared to those of previous years to identify if there has been an improvement in student grades. An analysis of factors that potentially influence these results in the two years has established comparability.

- Student Opinion. Survey methods can be used to address student motivation and acceptance. Combining open and closed questions provides a multi-dimensional view. The opinions of students can help us to evaluate the usability of the system. We asked students questions relating to the ease of use, navigation and course content. The questionnaire included asking students if they agreed or disagreed with a series of statements relating to the usability of the tutoring system, the tutoring system's course content and material, and their learning experience. The possible answers (on a Likert scale) were strongly agree, agree, undecided, disagree, or strongly disagree. Other questions involved students rating various given options, choosing a reply from a gradual scale, and answering questions in their own words. A random selection of 36 students out of 89 completed the questionnaire. This cohort is representative of the make-up of the entire class. The aspects gender, degree programme and previous experience with tutoring system 
were considered. Aspects such as age were neglected, as the class was very homogeneous in this respect.

- Student Behaviour. Student behaviour can be determined by examining Web access logs, which store details regarding the student's usage of the system. We used this information to validate the student performance results and to complement our survey data using Web usage mining (Pahl, 2004). We can evaluate the system by studying how the students used the system - when, how often, for how long, etc.

Together these methods allow us to assess the overall effectiveness and usability of the tutoring system in terms of the objectives outlined in the previous section.

\section{Evaluation results}

\subsection{Student performance}

The standard of the exam has remained constant over the last number of years. Over the course of the previous four years there has been a $2 \%$ increase per annum in the marks obtained by students in SQL exams as a result of gradual improvements and corrections to the system. The average SQL exam result obtained by last year's class was $56.9 \%$. The average mark this year was $62.3 \%$. This is an increase in one year of $5.4 \%$, a jump of over $3 \%$ of that which was expected.

\subsection{Student opinion}

The aspects that we looked at can be divided into three categories

- general usability,

- effectiveness in terms of feedback and guidance, active learning and training, in comparison to traditional approaches,

- general benefits and perceived strengths and weaknesses of the environment. Table 1 summarises the results of Likert-style questions related to the first two aspects. Probability measures based on a mapping of our Likert scale to ordinal values are added. We discuss the implications from specific mean and SD values in the discussion of the results below, if necessary. We have, however, not observed any unusual value distributions.

$<<$ Table 1 goes here $>>$

General usability:

- Question 1: The system is easy to use. A large majority of students, 75\%, agreed or strongly agreed that the system is easy to use. Close to $17 \%$ of those surveyed were undecided.

- Question 2: The system is easy to navigate. A majority of $63.9 \%$ of students agreed or strongly agreed that the system is easy to navigate. Approximately $22 \%$ were undecided, while the remainder felt that the system was not easy to navigate.

Effectiveness - Feedback, Guidance and Adaptivity:

- Question 3: The feedback element is useful. The feedback feature is an important part of the system. The survey results regarding its usefulness vary. $40 \%$ agreed (31.4\%) or strongly agreed (8.6\%) that it is useful. The same amount was 
undecided. Around 20\% did not think it was useful. While a significant proportion was neutral, the percentage that found it useful was double that who did not.

- Question 4: The guidance element is useful. A guidance feature based on a mid-tolong assessment is a unique part of our online tutoring system. Over two fifths of students agreed that this personalised guidance is useful. Slightly more than this amount was undecided.

- Question 5: Having adaptable levels of feedback and guidance is useful. The students were given the freedom to choose the levels of feedback and guidance they were given. Alternatively they could let the system decide the levels, which would be adapted according to the number of attempts the student has made for a particular question. A considerable majority of $72.2 \%$ agreed or strongly agreed that various levels of feedback and guidance are useful.

Effectiveness - Active Learning and Training:

- Question 6: The system is sufficient enough to allow the student to feel competent using SQL. Opinions on whether the system was sufficient enough to allow them to feel competent using SQL varied. 50\% agreed or strongly agreed that the system was sufficient. A quarter was undecided, while a quarter disagreed.

- Question 7: I enjoy learning SQL through practising them. A definite majority of $63 \%$ expressed their enjoyment in learning SQL by practising how to program. 28.6\% were undecided. Just 8.5\% disagreed.

Effectiveness - Comparison Automated and Traditional Tutoring:

- Question 8: Using the SQL tutor is more useful than attending lectures, tutorials and labs in preparation for continuous assessment and exams. Opinion was divided quite evenly as to whether the online tutoring system was more useful than attending lectures, tutorials and labs in preparation for the exams. Just over $30 \%$ agreed or strongly agreed with the above statement, practically the same percentage were undecided, while the same again disagreed.

- Question 9: The SQL tutor is a useful training and learning tool. The above question asked students if they felt the tutor was more useful than traditional methods of teaching and learning. A very significant majority of over $90 \%$ of students agreed, some strongly, that this was the case.

In addition to judging these statements comparing the traditional with this novel approach to tutoring, we also ask the students to rank the degree to which a tutoring system can replace a human tutor, at least partially or with respect to core functions:

- The tutoring system was used in conjunction with lectures and supervised lab sessions. We asked the students to indicate, on a scale of one (yes) to ten (no), if they "thought the tutoring system could act as a complete replacement for human lab tutors". The average result was number seven on the scale.

- A significant number of students indicated that they used the tutoring system to prepare for an exam. We asked the students whether they "feel that their exam results would be better because they used the system". A quarter answered with a definite "yes" (number one on the scale). The average result was number four on the scale.

Another aim was to identify benefits of the approach and the system that were most important to the students; see Table 2 . The students were given a list of five options (students could add further answers) and were asked to rate them from one 
(most important) to five (least important). The students could tick as many options as applied:

- Always available. Approximately $25 \%$ rated this advantage as being the most important of all options, with the same number rating it as the second most important. Close to a quarter (22.6\%) rated it as being the third most important.

- Self-paced learning. A rating of number two received the highest number of ticks (38.7\%) from those surveyed, followed by a number three rating at just over 25\%. The remaining votes were split almost evenly between the remaining ratings.

- Easy to use. 6.5\% rated the system's ease of use as being most important. Double this rated it as being least important. 22.5\%, 22.6\% and 35.5\% rated it as number two, three and four, respectively.

- A new and enjoyable way to learn. The highest ratings for this option had it at least important (45.2\%) and second least important (29\%).

- Practical rather than lecture based. A majority of almost $42 \%$ rated this option as the most important. Interestingly, this option also received the second highest rating (19.3\%) overall of "least important".

The main motivation for using the system was to prepare for the exam - over $45 \%$ indicated this was a reason for using it. Close to $30 \%$ indicated that they found it easy to use the tutoring system to learn SQL.

\section{$<<$ Table 2 goes here $>>$}

Finally, we asked the students to list strengths of the system and provide suggestions for further improvement - these are summarised below with percentage weightings given in brackets.

- The perceived strengths are easy to use (16.67), always available (16.67), helpful feedback (12.5), interactive (8.33), practical and activity based (12.5) and selfpaced (8.33) in terms of the benefit options.

- Students also suggested areas of the system that could be improved, including accuracy issues (17.38), input issues (syntax) (13.04), feedback quality (21.74) and quantity of questions to practise (13.04).

\subsection{Student behaviour}

We studied the behaviour of students from begin of term until the final exam.

- A little over $81 \%$ of the total usage of the system took place before a midsemester lab exam, while the remainder occurred after this exam until the final exam.

- $22.8 \%$ of usage before the mid-semester exam occurred during the scheduled lab sessions, which took place once a week for two hours at a time. Human lab assistant were available for consultation during these sessions.

- $77.2 \%$ of the pre-exam usage took place outside of these sessions, some on college computers and some from elsewhere (mostly home or workplace). Almost $28 \%$ of the total pre-lab exam usage occurred after six o'clock in the evening. Weekend usage accounted for $4.5 \%$ of this period of usage.

- Post mid-semester exam, almost $11 \%$ of usage took place during the scheduled lab sessions. The remainder took occurred on weekdays outside of scheduled lab sessions, one third of which was after six o'clock in the evening. 


\section{Discussion}

\subsection{Student performance}

The introduction of the new SQL tutoring system has improved student performance in the SQL exam. The trend over the last number of years was for the average exam mark to increase by $2 \%$ on the year previously. When our tutoring system was introduced, it was the only change in the module, the system and in the traditional classroom-based setting; all other factors, including the difficulty of the SQL examinations, remained constant. The average exam result has increased by $5.4 \%$ on last year's average mark.

\subsection{Student opinion - usability of the tutoring system}

A guided discovery learning environment was a key consideration in the design of the SQL tutoring system. Students can choose the direction of their own learning by choosing the combination of questions they wanted to try. This implies that the navigation of the system that implements the guidance is a basic, but central feature, which is a prerequisite for the more advanced features. The majority of students agreed or strongly agreed that the system based on guided discovery is easy to use.

\subsection{Student opinion - feedback and guidance}

The students' attitude towards feedback and guidance is characterised by low levels of tolerance of inaccuracies in correction and feedback, but also a high appreciation of learner-controlled adaptivity in both feedback and guidance elements.

The feedback and personalised guidance features are an important part of our tutoring system. Indeed, a personalised guidance feature based on a mid-to-long term assessment is a unique element in SQL tutoring systems. The survey results regarding the usefulness of these features are overall positive, but also vary to some extent, which points to a very important observation. While the percentage that found it useful was double that who did not, the discussion we had with students and suggestions for improvements indicate that occasional inaccuracies of the correction component that analyses student submission have impacted this result negatively. Students only accept automated feedback and guidance based on the feedback analysis, if a high degree of accuracy is achieved.

Any apprenticeship model contains a stage where the student is given advice and help when and if s/he needs it. Based on the virtual apprenticeship approach (Murray, Ryan, \& Pahl, 2003), which is an adaptation of the cognitive apprenticeship model (Collins et al., 1991) to virtual learning environments, we provided a method of allowing the student to tailor the levels of feedback or guidance. A considerable majority agreed that it is useful to have scaffolding and to be able to influence its levels of support.

\subsection{Student opinion - active learning}

The primary aim of this tutoring system is to help students to learn how to create SQL statements and train problem solving in this context. Very positive results were 
found with a significant majority indicating that the tutor has improved their understanding of SQL as a database programming language. Activity-based learning is a key part of this achievement. Students learn by practising how to program in SQL rather than learning about various methods of creating SQL statements from a textbook. Over $90 \%$ agreed, some strongly, that the tutoring system is a useful training and learning tool in its own right.

Ideally, for the use of the system to be a positive learning experience, the students should enjoy their learning and training activities. A definite majority expressed their enjoyment of learning select statements through an active learning approach by practising them.

\subsection{Student opinion - comparison}

Online tutoring systems are often developed to replace the traditional classroombased teaching and learning environment, in which a human tutor would be present. Our tutoring system was used voluntarily in conjunction with traditional lectures and timetabled lab sessions. This blended learning approach provides a combination of computer-based and human-presented learning.

Opinion was split amongst the students as to whether the online tutoring system was more useful than attending lectures, tutorials and labs in preparation for the aforementioned assessments. Votes were divided evenly between those who agreed in some manner, those who were undecided, and those who disagreed. Considering that the question asked if the tutoring system could act as a complete replacement, this demonstrates that students are open to have at least some functions of a human tutor replaced by an automated one. In our case the automated tutor was actually accepted as equally suitable as a human tutor for the purpose of exam preparation. The disadvantages of inaccuracy and social detachment are outweighed by the perceived benefits.

\subsection{Student opinion - benefits and weaknesses}

Students indicated that the most important aspect of the system is that it is practical rather than lecture based. This supports our hypothesis of active learning and training as a beneficial setting. The second most important aspect is that the tutoring system is always available, which can in practice only be provided through automated knowledge-level feedback and guidance.

A significant number of students indicated that they used the tutoring system to prepare for exams. When asked to grade their thoughts on whether their exam results would be better by using the system, three out of four students gave a positive response, i.e. that they felt using the system would result in better results for them in the SQL exam. This confirms the hypothesis that an automated feedback system is accepted at least as an adequate learning tool in comparison with human tutorsupported lab sessions.

Students also suggested areas of the system that could be improved. While some minor aspect such as insufficient explanations and hints can easily be dealt with, the students have pointed to the main challenge of an automated analysis and correction 
technique for student submissions. It is very difficult to achieve an accurate correction method that is infallible. Inaccuracy leads to frustration on the students' side. Almost immediate intervention of staff was required in this case. For languages of the complexity of SQL, it seems however possible based on our and other researchers' experience and to provide an acceptable degree of accuracy (Mitrovic \& Ohlsson, 1999; Kenny, 2006).

\subsection{Student behaviour}

The information found through Web usage mining can tell us about the behaviour of students interacting with the (Web-based) tutoring system. We used this technique to validate and complement the results of both the student performance evaluation and student opinion evaluation. We examined usage of the system before and after a midsemester lab exam on SQL took place until the final exam. Over four fifths of the total usage happened before the mid-semester exam. This was probably because SQL was covered in the lectures in the weeks preceding the mid-semester lab exam, and because students were preparing for this programming oriented lab exam.

Web usage mining verifies the students' own assessment of $23 \%$ of the pre-exam usage of the system during lab sessions. Students said that they used the system to prepare for a mid-semester SQL exam. Generally, a human tutor would not have been available during evening or early morning hours when the system was frequently used. Although we have observed "just-in-time" learning close to exams, we can also deduce through Web mining that this is complemented by long-term and pre-emptive use. The majority of usage occurred outside of the scheduled lab sessions. Most students used the system at college, but some did access it externally.

\section{Conclusions}

Automated tutoring has become an acceptable method of instruction in universities and institutions. The implementation of such systems must be coupled with sound teaching and learning methods. Learning is often divided into two categories knowledge acquisition and skills training. While the types are different, students reach a higher level of understanding when being actively engaged. There should be a high level of knowledge-level and skills-level interaction between the student and a tutoring system. An important feature of the apprenticeship theory is scaffolding, in particular feedback. Feedback can be global or local, synchronous or asynchronous. Any automated tutoring system should offer synchronous local feedback when errors have been made, as well as providing global feedback in the form of guidance after a period of time based on the student's overall performance. Indeed, we see automated local and global feedback as a prerequisite for an effective online active learning environment.

Adaptivity and a realistic setting are further success factors in online learning (Murray et al., 2003). The student should have control over her/his learning experience. The reasons for this student autonomy are two-fold: firstly, it encourages a sense of responsibility; and secondly, this empowerment increases the motivation levels. This has led to facilitative individually tailored adaptive learning environments. Adaptivity in the system is managed by the guidance and 
recommendation component, controlled by the student. A realistic setting has longterm benefits. By teaching students a topic in an environment similar to the real one, students will learn from the very beginning how to apply skills in the proper context.

Scaffolding in the form of immediate, synchronous feedback and guidance at knowledge-level is a key feature of a successful online tutoring system. The system's accuracy and the student's trust level are also important for its success. We evaluated a tutoring system along three lines: student performance, student opinion, and student behaviour. The introduction of the tutoring system has improved student performance in exams. While students have generally seen the benefits of the system, certain features were subject to mixed opinions. For example, although generally positive, opinion varied on the usefulness of the feedback and guidance features and the extent to which a tutoring system could act as a replacement for a human tutor. A significant number of these concerns can be attributed to the technical issue of accuracy of analysis and corrections, which turns out the main technical challenge. However, even though tolerance levels are low, we feel that these problems can be rectified.

Designing and implementing a flawless correction method in general is a complex task. Students' trust in the system will increase as correction errors are lessened; they will be confident in relying on the approach if their errors are accurately diagnosed and the scaffolding provided is relevant. This can be achieved for a problem setting such as database programming, as our and other researchers' experience has demonstrated. This raises the question of transferability of our results to other subjects. Formal languages lend themselves to online tutoring - their structure and description in terms of grammar and semantic definitions makes them relatively easy to analyse. This includes textual and graphical languages, to which at least Web-based learning and training environments would anyway be limited by electronic data representations. The student can make submissions to the system and receive results based on a correction method that considers grammar and semantic rules. The only limitation is the effort required to provide an acceptably accurate solution.

\section{References}

Brusilovsky, P. (2000). Adaptive Hypermedia: From Intelligent Tutoring Systems to Web-based education. Proceedings of 5th International Conference on Intelligent Tutoring Systems, ITS 2000. 1-7.

Chou, C.-Y., Chan, T.-W., \& Lin, C.-J. (2002). Redefining the learning companion: the past, present, and future of educational agents. Computers \& Education, 40 (3), 255-26.

Collins, A., Brown, J.S., \& Holum, A. (1991). Cognitive Apprenticeship: Making thinking visible. American Educator, Winter edition.

De Bra, P., Brusilovsky, P., \& Houben, G. (1999). Adaptive hypermedia: from systems to framework. ACM Computing Surveys, 31(4), Article 12.

Engeström, Y. (1987). Learning by expanding: An activity-theoretical approach to developmental research. Helsinki: Orienta-Konsultit. 
Grabinger, R.S. (1996). Rich environments for active learning. In D. H. Jonassen (ed): Handbook of research for educational communications and technology. New York: Macmillan.

Guzdial, M. \& Kehoe, C. (1998). Apprenticeship-based learning environments: A principled approach to providing software-realized scaffolding through hypermedia. Journal of Interactive Learning Research, 9(3/4), 289-336.

Hartley, J. R. (1973). The Design and Evaluation of an Adaptive Teaching System. International Journal of Man-Machine Studies, 5(3), 421-436.

Herrington, J. \& Oliver, R. (2000). An instructional design framework for authentic learning environments. Educational Technology Research and Development, 48 (3), 23-48.

Kenny, C. (2006). Automated Tutoring for a Database Skills Training Environment. M.Sc. Thesis. Dublin City University, School of Computing.

Kinshuk, Patel, A., \& Russell, D. (2000). A Multi-institutional Evaluation of Intelligent Tutoring Tools in Numeric Disciplines. Educational Technology \& Society $3(4)$.

Laurillard, D. (1993). Rethinking University Teaching. London: Routledge.

Melis, E. \& Ullrich, C. (2003). Local and global feedback. Proceedings of AIED2003, $11^{\text {th }}$ International Conference in Artificial Intelligence in Education.

Mitrovic, A. \& Ohlsson, S. (1999). Evaluation of a Constraint-Based tutor for a database language. International Journal of Artificial Intelligence in Education, 10, 238-256.

Murray, S., Ryan, J., \& Pahl, C. (2003). A Tool-mediated Cognitive Apprenticeship Approach for a Computer Engineering Course. International Conference on Advanced Learning Technologies ICALT'2003, 2-6. IEEE.

Murray, T. (1999). Authoring Intelligent Tutoring Systems: An analysis of the state of the art. International Journal of Artificial Intelligence in Education, 10, 98-129. 1999.

Nardi, B. (ed.) (1997). Educational Context and Consciousness: Activity Theory and Human-Computer Interaction. MIT Press.

Pahl, C. (2004). Data Mining Technology for the Evaluation of Learning Content Interaction. International Journal on E-Learning IJEL. 3(4), 47-55. AACE.

Patel, A., Scott, B., \& Kinshuk. (2001). Intelligent tutoring: from SAKI to Byzantinum. Kybernetes, 30(5/6), 807-818. 
Ravenscroft, A., Tait, K., \& Hughes, I. (1998). Beyond the Media: Knowledge Level Interaction and Guided Integration for CBL Systems. Computers \& Education, 30(1/2), 49-56.

Scott, B. (2001). Conversation Theory: A Constructivist, Dialogical Approach to Educational Technology. Cybernetics \& Human Knowing, 8(4), 25-46.

Winnips, K. (2001). Scaffolding-by-design as a model for online learner support. Ph.D. thesis, Faculty of Educational Science and Technology, University of Twente. 


\section{Captions}

Figure 1. Interactive SQL tutoring environment.

Table 1. Combined results of Likert scale questions.

Table 2. system benefits rating. 
Figure 1

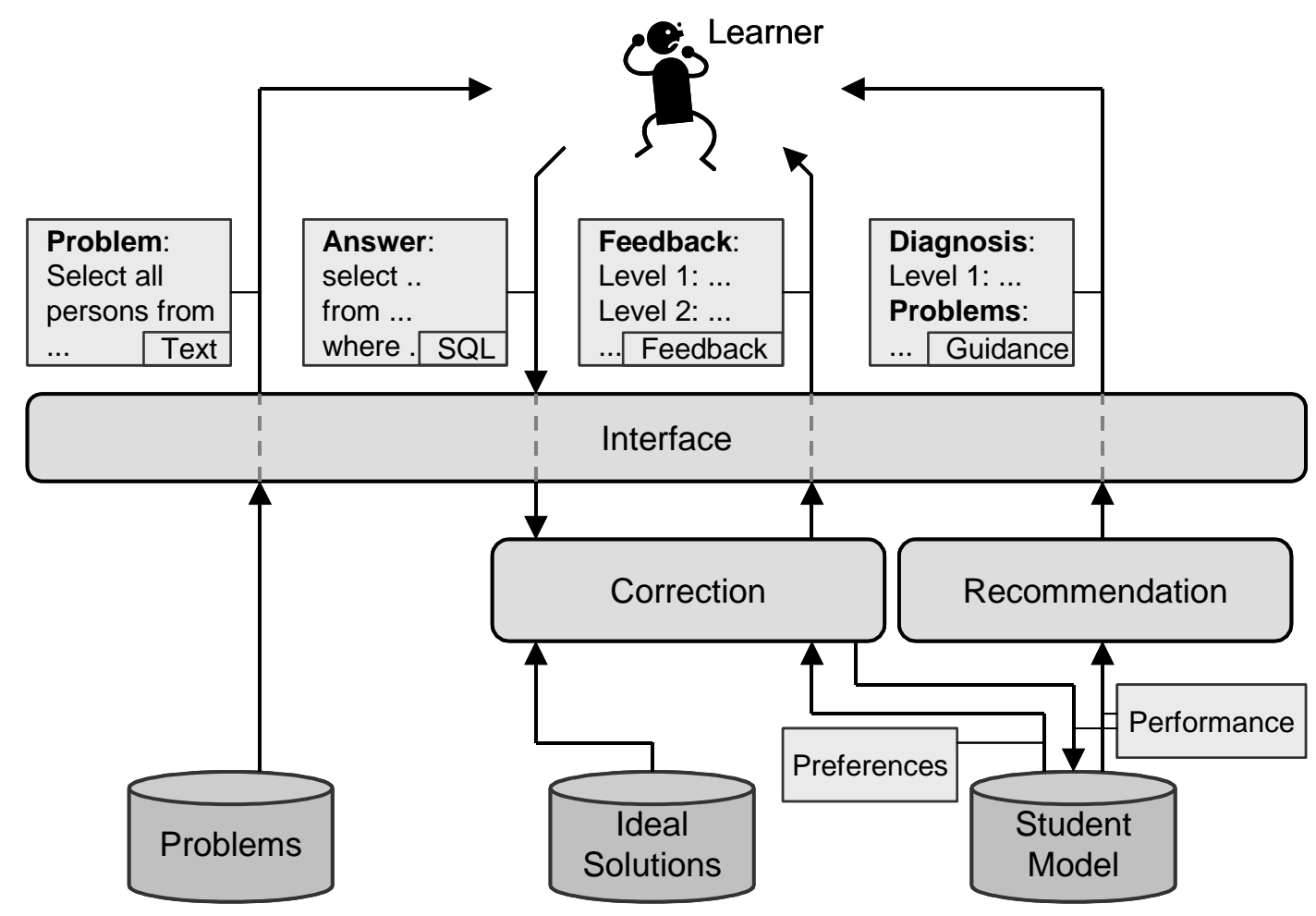


Table 1

\begin{tabular}{|l|r|r|r|r|r|r|r|}
\hline \multicolumn{1}{|c|}{ Question number } & $\begin{array}{c}\text { strongly } \\
\text { agree } \\
(=2)\end{array}$ & \multicolumn{1}{c|}{ agree } & $\begin{array}{c}\text { un- } \\
\text { decided } \\
(=0)\end{array}$ & $\begin{array}{c}\text { dis- } \\
\text { agree } \\
(=-1)\end{array}$ & $\begin{array}{c}\text { strongly } \\
\text { disagree } \\
(=-2)\end{array}$ & mean & SD \\
\hline 1 (ease of use) & 13.9 & 61.1 & 16.7 & 8.3 & 0 & 0.97 & 0.82 \\
\hline 2 (easy to navigate) & 13.9 & 50 & 22.2 & 11.1 & 2.8 & 0.83 & 1.02 \\
\hline 3 (feedback useful) & 8.6 & 31.4 & 40 & 20 & 0 & 0.69 & 0.94 \\
\hline 4 (guidance useful) & 0 & 44.4 & 47.3 & 8.3 & 0 & 0.83 & 0.64 \\
\hline 5 (adaptivity useful) & 2.8 & 69.4 & 25 & 2.8 & 0 & 0.97 & 0.62 \\
\hline 6 (sufficiency) & 2.8 & 47.2 & 25 & 22.2 & 2.8 & 0.5 & 0.97 \\
\hline 7 (enjoyment) & 20 & 42.9 & 28.6 & 8.5 & 0 & 1.03 & 0.9 \\
\hline 8 (comparison) & 5.7 & 25.7 & 31.5 & 31.5 & 5.6 & 0.26 & 1.12 \\
\hline 9 (usefulness) & 17.2 & 74.3 & 5.7 & 0 & 2.8 & 1.09 & 0.78 \\
\hline
\end{tabular}


Table 2

\begin{tabular}{|l|l|l|l|l|l|}
\hline & \multicolumn{1}{|c|}{ Rate 1\% } & \multicolumn{1}{|c|}{ Rate 2\% } & Rate 3\% & Rate 4\% & \multicolumn{1}{|c|}{ Rate 5\% } \\
\hline Always available & 25.8 & 25.8 & 22.6 & 12.9 & 12.9 \\
\hline $\begin{array}{l}\text { Self-paced } \\
\text { learning }\end{array}$ & 12.9 & 38.7 & 25.7 & 12.9 & 9.7 \\
\hline Easy to use & 6.5 & 22.5 & 22.6 & 35.5 & 12.9 \\
\hline $\begin{array}{l}\text { New and enjoyable } \\
\text { way to learn }\end{array}$ & 12.9 & 6.5 & 6.5 & 29 & 45.2 \\
\hline $\begin{array}{l}\text { Practical rather } \\
\text { than lecture based }\end{array}$ & 41.9 & 6.5 & 22.6 & 9.7 & 19.3 \\
\hline
\end{tabular}

